\title{
Classification of Mild Cognitive Impairment and Alzheimer Disease Using Model-Based MR and Magnetization Transfer Imaging
}

\author{
R. Wiest, Y. Burren, M. Hauf, G. Schroth, J. Pruessner, M. Zbinden, K. Cattapan-Ludewig, and C. Kiefer
}

\begin{abstract}
BACKGROUND AND PURPOSE: Early stratification of degenerative processes is a prerequisite to warrant therapeutic options in prodromal Alzheimer disease. Our aim was to investigate differences in cerebral macromolecular tissue composition between patients with AD, mild cognitive impairment, and age- and sex-matched healthy controls by using model-based magnetization transfer with a binary spin-bath magnetization transfer model and magnetization transfer ratio at 1.5T.
\end{abstract}

MATERIALS AND METHODS: We investigated patients with de novo AD $(n=18), \mathrm{MCl}(n=18)$, and CTRLs $(n=18)$. A region-of-interest analysis of the entorhinal cortex, hippocampal head and body, insula, and temporal neocortex was performed with fuzzy clustering to associate every subregion to a cluster representative for each group.

RESULTS: Cluster analysis achieved a concordance of 0.92 (50 of 54 subjects) between a combination of the calculated mMT parameters ( $k f, k r, T 2 r, F, T 2 f)$ in the entorhinal cortex and the neuropsychological diagnosis. The sensitivity and specificity for the discrimination of AD from $\mathrm{MCl}$ reached 1 and 0.94 , with a positive predictive value of 0.95 and a negative predictive value of 1 . Compared with mMT, the concordance for MTR was 0.83 (45 of 54 subjects) with a lower specificity of 0.5 and positive predictive value of 0.67 to discriminate patients with $\mathrm{AD}$ and $\mathrm{MCl}$.

CONCLUSIONS: $\mathrm{mMT}$ imaging detects macromolecule-related alterations and allows an improved classification of patients with early AD and $\mathrm{MCl}$ compared with MTR.

ABBREVIATIONS: $A D=$ Alzheimer disease; $C E R A D-N A B=$ German Version of the Consortium to Establish a Registry on Alzheimer's Disease-Neuropsychological Assessment Battery; $\mathrm{Cl}$ = confidence interval; CTRLs = healthy controls; $\mathrm{MCl}=$ mild cognitive impairment; $\mathrm{mMT}$ = model-based magnetization transfer; $\mathrm{MTR}=$ magnetization transfer ratio

maging biomarkers for early diagnosis of neurodegenerative disorders are being increasingly recognized as important arrays in a diagnostic framework to support clinical findings of cognitive decline. Beyond analysis of amyloid $\beta$ and $\tau$ species in CSF, ${ }^{1}$ in vivo analysis of the degree of cerebral atrophy during earlier

\footnotetext{
Received May 15, 2012; accepted after revision June 21.

From the Support Center for Advanced Neuroimaging (R.W., Y.B., M.H., G.S., M.Z., C.K.), University Institute of Diagnostic and Interventional Neuroradiology, Inselspital, University of Bern, Bern, Switzerland; Sanatorium Kilchberg, Psychiatric Private Hospital (K.C.-L.), Kilchberg/Zurich, Switzerland; University Hospital of Psychiatry Bern (Y.B., K.C.-L.), Bern, Switzerland; and McGill University Centre for Studies in Aging, Neurology, and Neurosurgery (J.P.), McGill University, Montreal, Quebec, Canada.

This work was supported by the Kamillo-Eisner Foundation, grant No. EK 145/05. Please address correspondence to Claus Kiefer, PhD, Support Center for Advanced Neuroimaging, University Institute of Diagnostic and Interventional Neuroradiology, Inselspital, University of Bern, Freiburgstr, CH-3010 Bern, Switzerland, Tel; e-mail: claus.kiefer@insel.ch

Indicates article with supplemental on-line appendix

Evidence-Based Medicine Level 2.

http://dx.doi.org/10.3174/ajnr.A3307
}

stages of AD is very important to initiate therapies ahead of irreversible brain damage. ${ }^{2}$ Novel MR imaging-based strategies aiming toward individual classification analyses are increasingly investigated to identify de novo patients in a routine clinical setting. ${ }^{3}$ Significant atrophy that accompanies the conversion from mild cognitive impairment to Alzheimer disease is most likely to occur in the mesial and inferior temporal lobes and temporoparietal and frontal neocortices. ${ }^{4-6}$ The Alzheimer Disease Neuroimaging Initiative work demonstrated that computational neuroanatomic methods, including automated classifiers, can be successfully applied to quantify local patterns of brain atrophy in cognitively healthy individuals and in patients with prodromal or mild AD. ${ }^{7}$ The neurodegenerative component of $\mathrm{AD}$ has been demonstrated to be the direct substrate of cognitive impairment; thus, molecular biomarkers of neuronal injury that are present in advance of atrophy offer a complementary target for MR imaging. ${ }^{8}$

To explore neurodegenerative processes in a clinical environment along with the pathophysiologic effects related to macromo- 
Demographic data, MR imaging gray matter/total intracranial volume ratio, Mini-Mental State Examination, and neuropsychological test (CERAD-NAB) parameters

\begin{tabular}{|c|c|c|c|c|c|c|}
\hline Characteristics & Control Mean & Control SD & MCI Mean & MCI SD & AD Mean & AD SD \\
\hline No. & 18 & & 18 & & 18 & \\
\hline Age (yr) & 71.61 & 9.2 & 70.83 & 10.1 & 70.39 & 9.9 \\
\hline Education & 12.78 & 3.11 & 11.28 & 1.99 & 11.00 & 3.30 \\
\hline Men/women & $9: 9$ & & 9:9 & & 9:9 & \\
\hline MRI TIV/GM ratio (GM fraction) & 2.61 & 0.28 & 2.82 & 0.42 & 2.86 & 0.43 \\
\hline MMSE (30) & 29.50 & 0.7 & 28.67 & 1.49 & $24.56^{b}$ & 3.45 \\
\hline \multicolumn{7}{|l|}{ CERAD } \\
\hline Verbal fluency $(24)^{c}$ & 24.89 & 7.28 & $16.06^{b}$ & 5.07 & $10.44^{b}$ & 3.88 \\
\hline Modified BNT (15) & 14.56 & 0.78 & 14.50 & 0.99 & $12.33^{\mathrm{b}}$ & 2.50 \\
\hline Word list: delayed recall (10) & 8.28 & 1.60 & $6.50^{\mathrm{b}}$ & 2.30 & $2.33^{b}$ & 1.45 \\
\hline Word list: learning (30) & 22.22 & 3.06 & 19.61 & 4.27 & $12.94^{\mathrm{b}}$ & 5.83 \\
\hline Constructional praxis (11) & 10.78 & 0.95 & 10.56 & 0.78 & $9.39^{b}$ & 1.91 \\
\hline Constructional praxis: delayed recall (11) & 9.83 & 2.15 & 9.39 & 2.06 & $4.28^{\mathrm{b}}$ & 3.44 \\
\hline CERAD total score (101) & 90.56 & 15.82 & $76.62^{b}$ & 15.47 & $51.71^{b}$ & 19.01 \\
\hline
\end{tabular}

Note:-BNT indicates Boston Naming Test; TIV, total intracranial volume; GM, gray matter; MMSE, Mini-Mental State Examination.

${ }^{a}$ Maximum scores of test subunits are in brackets

${ }^{b}$ One-way ANOVA $(P<.05)$

`Verbal fluency does not have a ceiling when administered using standard instructions as part of the CERAD. For calculation, a cap of 24 was placed on verbal fluency, which represents 1 SD above the normal aging population mean.

lecular deposition, an imaging technology that determines physical properties of the brain tissue, which reflect the exchange of magnetization between molecules of water and molecules of more-solid structural components, may close the gap between $\tau$-mediated neuronal injury and atrophy. Several studies that incorporated magnetization transfer ratio revealed a widespread distribution of tissue changes in $\mathrm{AD}$ and $\mathrm{MCI}$ in the temporal lobe before gross structural changes became apparent ${ }^{9,10}$ and a progressive MTR reduction with time. ${ }^{11}$ While MTR is basically a phenomenologic measure that has been shown to depend both on the amount of magnetization transfer and the direct saturation of free water by the radio-frequency pulse, ${ }^{12}$ model-based magnetization transfer imaging allows a more comprehensive analysis by investigating the exchange of magnetization between molecules of water and molecules of more solid structural components more in detail.

Model-based quantification in general ${ }^{13,14}$ is an approach favored in an increasing number of centers to become independent of hardware technology. A methodologic study using mMT that aimed at an automated fuzzy-c-means based classification of cognitively normal young and elderly individuals, MCI and AD patients with respect to hippocampal subregions, reported a sensitivity of $69 \% .^{15}$ Beyond the hippocampus, a recent whole-brain group analysis using mMT confined to gray matter regions detected significantly reduced efficiency of the transfer of magnetization between the 2 pools in the posterior cingulate and posterior parietal cortex of patients with $\mathrm{AD}$ compared with healthy controls. ${ }^{16}$ To optimize mMT-based categorization of patients with $\mathrm{AD}$ and MCI, our study aimed to investigate an improved technique for automated classification of individual patients that incorporated a VOI analysis of brain areas where a high presence of accumulated macromolecules was expected. We hypothesized the following: 1) that mMT imaging would improve the detection of macromolecular-related changes in cortical subregions commonly affected during MCI and early AD compared with MTR, and 2) that automated classification of mMT allows a stratification of individuals with memory symptoms when admitted for the first time to a memory clinic.

\section{METHODS AND MATERIALS \\ Subjects}

All subjects were de novo patients admitted for the first time to a memory clinic due to memory problems. The clinical examinations were performed at the outpatient clinic of our institution (Support Center for Advanced Neuroimaging, University Institute of Diagnostic and Interventional Neuroradiology, Inselspital, University of Bern) by a board-certified psychiatrist and neuropsychologist. The study was approved by the ethics committee of the hospital medical faculty, and all study participants gave informed written consent. The following subjects were included in the study: 18 individuals fulfilling the clinical criteria of mild-tomoderate $\mathrm{AD}$, as established by the National Institute of Neurologic and Communicative Diseases and Stroke/Alzheimer Disease and Related Disorders Association ${ }^{17}$; and 18 individuals fulfilling the criteria of MCI. ${ }^{18}$ Eighteen healthy control individuals were recruited from the local educational program for older people. All subjects were prospectively divided on first referral according to age and sex, by using a matched-pair design. Their neuropsychological functions were tested by the German Version of the Consortium to Establish a Registry on Alzheimer Disease-Neuropsychological Assessment Battery (Table). ${ }^{19}$ Patient inclusion criteria were unexplained progressive memory deficits, as described by the International Classification of Diseases (http://www.who.int/ classifications/icd/en/GRNBOOK.pdf) for dementia. Patients were classified as having probable $\mathrm{AD}$ if they presented with an amnestic syndrome, including impairment in learning and recalling recently learned information, and additional evidence of cognitive dysfunction in at least 1 other cognitive domain. ${ }^{20} \mathrm{~A}$ diagnostic routine $\mathrm{MR}$ imaging was performed to exclude other treatable causes of cognitive decline (ie, vascular dementia, normal pressure hydrocephalus, or brain tumor) within 4 weeks after the cognitive testing.

\section{Structural MR Imaging}

Structural MR imaging was performed on a $1.5 \mathrm{~T}$ MR imaging scanner (Magnetom Vision-Sonata; Siemens, Erlangen, Germany). MR imaging included a T1-weighted, sagittally oriented 
3D magnetization-prepared rapid acquisition of gradient echo sequence (TR/TE/TI, 2000/3.42/1100 ms; matrix, $256 \times 25$; FOV, $256 \times 256 \mathrm{~mm}$; flip angle, $15^{\circ}$; slab, $160 \mathrm{~mm}$ ) with a $1-\mathrm{mm}^{3}$ isovoxel resolution) and an axial FLAIR sequence (TR/TE/TI, 9130/117/2500 ms; 24 sections; 5-mm section thickness; gap, 1 mm; 2 averages; 2 concatenations; matrix, $205 \times 256$; bandwidth, $199 \mathrm{~Hz} /$ pixel, transversal).

\section{mMT}

The binary spin-bath magnetization transfer model ${ }^{13}$ enables separating effects related to a direct saturation of the free pool and real magnetization transfer effects and determining the fundamental physical parameters that characterize the shape of the magnetization transfer spectra: the relative size of the restricted proton pool (F), the magnetization exchange rates between the free and the restricted pools $(\mathrm{kf}$ [free $\rightarrow$ restricted], $\mathrm{kr}$ [restricted $\rightarrow$ free] $)$, the T2 relaxation time of the restricted pool (T2r), and the relaxation times T1 and T2 $f$ of the free pool, which were determined in separate experiments on the basis of the Bloch equations. $^{21-24}$ The restricted-to-free proton pool size ratio $(\mathrm{F})$ quantifies the macromolecular over free proton content within an individual voxel. The exchange rates (kf, $\mathrm{kr}$ ) express the throughspace transfer of magnetization between the reservoirs by magnetic dipole-dipole coupling and chemical exchange. The T2r relaxation time of the restricted pool can be interpreted as a marker for the type of the macromolecules and its coupling to the environment. The experimental base for the quantification of the magnetization transfer effects is a repeated presaturation of the sample with Gaussian radio-frequency pulses irradiated at different off-resonant frequencies. The magnetization transfer ratio was calculated by relating the magnetization transfer images acquired at $\Delta \mathrm{f}=1.00 \mathrm{kHz}$ to the data without presaturation (M0): $\mathrm{MTR}=100 \times[\mathrm{M} 0-\mathrm{mol} / \mathrm{L}(\Delta \mathrm{f}=1.0)] / \mathrm{M} 0$. The nonlinear fitting of the theoretic magnetization transfer model-based signal to the measured magnetization transfer signal intensity within each imaging voxel was accomplished by using a Levenberg-Marquardt algorithm, which provides the optimal parameter values for each voxel in a least-squares sense. In this way, complete new images are calculated that represent the different parameter values for each voxel. The magnetization transfer-weighted images were acquired by using a set of 7 gradient-echo FLASH sequences with Gaussian-modulated presaturation pulses at frequency offsets $(\Delta \mathrm{f})$ of $0.25,0.50,1.00,2.00,4.00,8.00$, and $16.00 \mathrm{kHz}$, according to the central $1 \mathrm{H}$ Larmor frequency $(\mathrm{TR}=300 \mathrm{~ms}, \mathrm{TE}=$ $4.18 \mathrm{~ms}, \alpha_{\mathrm{EXC}}=20^{\circ}, \alpha_{\mathrm{MT}}=540^{\circ}$ [Gaussian], section thickness $=$ $4 \mathrm{~mm}$ [gap 0], 16 sections [interleaved], FOV $=256 \mathrm{~mm}, 128 \times$ 128 matrix [data interpolated to $256 \times 256$ ]). The experiments were performed on a $1.5 \mathrm{~T}$ whole-body scanner. The acquisition time for the entire protocol (3D-T1, DTI, mMT) approximated 30 minutes.

\section{Volume-of-Interest-Based Analysis of the mMT Dataset}

Previous imaging-pathologic correlations have demonstrated that manual segmentation is a valid marker of neurodegenerative changes in $\mathrm{AD} .{ }^{25} \mathrm{We}$ focused on the area with the earliest patho- logic lesion formation - the temporal cortex, including the entorhinal cortex (EC), hippocampal head and body, insula, and inferior and middle temporal neocortices (TP) (Fig 1). The candidate volumes were selected on the basis of previous MR imaging and FDG-PET findings. ${ }^{26-28}$ To project these regions from a reference data template (Fig 1) in Montreal Neurological Institute space (SPM Anatomy toolbox, colin27T1; http:// www2.fz-juelich.de/inm/index.php?index =194) into the individual brains, the SPM5 (Wellcome Department of Imaging Neuroscience, London, UK) warping algorithms and matrices were used. Details of the segmentation procedure and data extraction are provided as supplementary material (On-line Appendix).

To determine the contribution of the magnetization transfer parameters MTR, T2r, F, kr, kf, the relaxation time of the free water pool, and the combined subset of the mMT parameters without (kf, kr, T2r, F) and with the relaxation time of the free water pool (kf, kr, T2r, F, T2f) to the classification of AD, MCI and CTRLs, we analyzed how subjects were classified within the different subregions by the magnetization transfer parameters, the mMT subsets, and a combination of all VOIs. Eight VOI-specific parameters were incorporated into the classification of the 5 VOIs in every hemisphere: MTR, T2r, T2f, F, kr, kf and 2 subsets (subset $1: \mathrm{kf}, \mathrm{kr}, \mathrm{T} 2 \mathrm{r}, \mathrm{F}$ and subset $2 \mathrm{kf}, \mathrm{kr}, \mathrm{T} 2 \mathrm{r}$, F, T2f). To avoid a bias due to local atrophy effects in the classification, we performed regional averaging of parameter values by using histograms with a normalized number of voxels within each region ${ }^{12}$ : If $f_{k}$ are the count numbers for each bin $\mathrm{k}$ and $\mathrm{f}_{\text {sum }}$ is the sum over all counts, the average value was calculated after weighting the histogram by $1 / \mathrm{f}_{\text {sum }}$. To quantify the correlation between the parameters of the free and restricted pool and especially to verify the negative correlation of T2f and T2r, we performed bootstrap analyses. ${ }^{15}$

Bootstrapping involves the generation of multiple versions of the cohort stratification, serving to ensure maximum learning efficiency from a limited dataset, and involves the generation of several random samples with replacement. The classification was performed with a Gustafson-Kessel algorithm. ${ }^{29}$ The GustafsonKessel algorithm associates each cluster with both a point and a matrix, representing the cluster center and its covariance (Fig 1, graphically illustrated for a single mMT parameter: T2r). This technique is capable of detecting hyperellipsoidal clusters of different sizes and orientations by adjusting the covariance matrix of data, thus overcoming the drawbacks of a conventional fuzzy-cmeans algorithm. This choice is essential because it makes the classification more robust against outliers and noise. The program for the cluster analysis is an in-house-written software based on the Matlab (MathWorks, Natick, Massachusetts) environment. ${ }^{15,30}$ The postprocessing procedure is fully automated and needs an estimated time of 60 minutes per patient. The notion "selectivity" is used throughout the article to express the correlation of the 2 classifications resulting from the neuropsychological and mMT evaluations.

A 1-way ANOVA was performed to test for differences between the neuropsychological parameters (Statistical Package for the Social Sciences, Version 11.5; SPSS, Chicago, Illinois). 


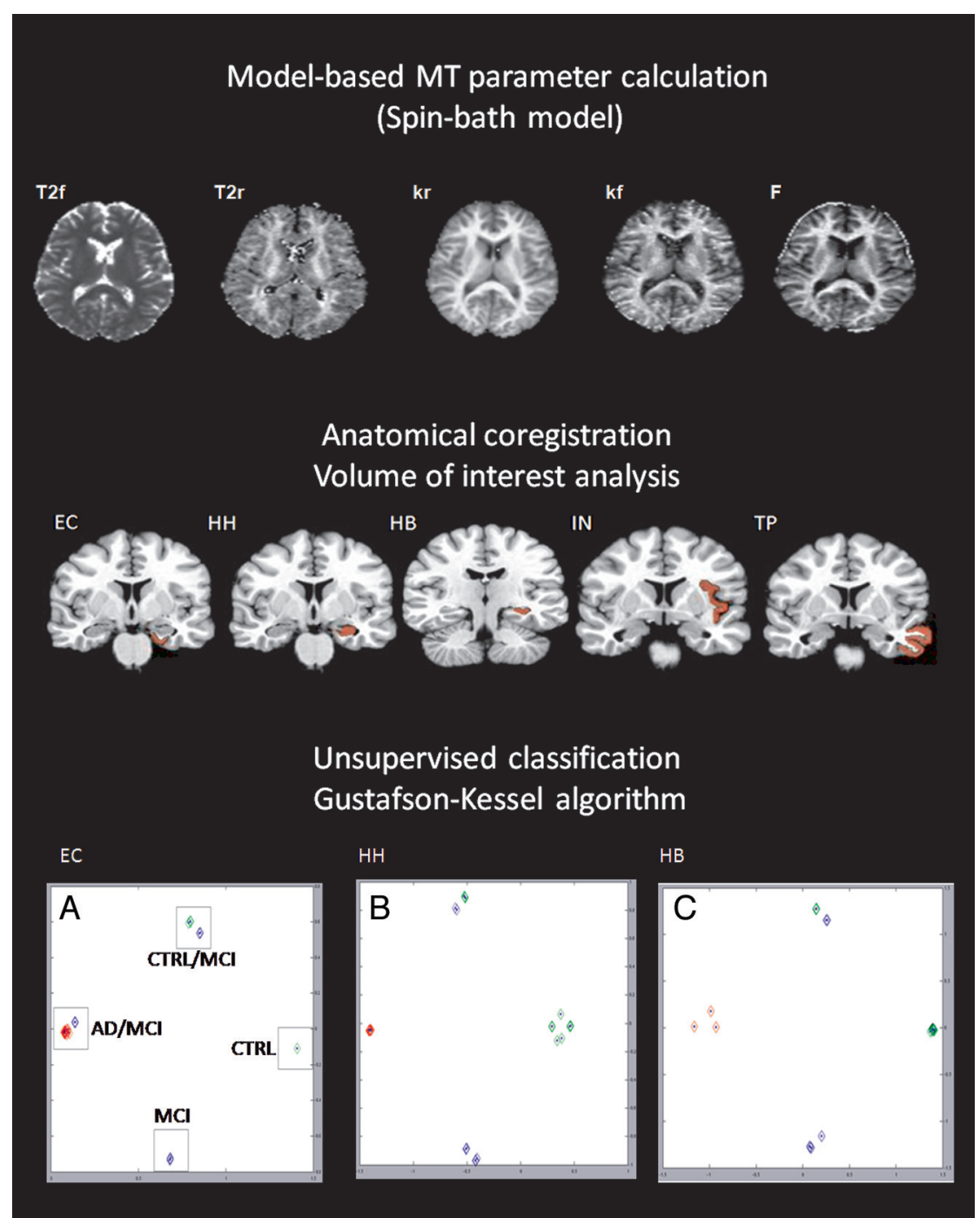

FIG 1. Exemplary graphic illustration of the clustering procedure (for T2r). Upper row: Representative model-based magnetization transfer parameter maps used for further classification. Middle row: After anatomic transformation of the 3D T1 template on the magnetization transfer parameter maps, the mMT values are extracted from the VOls highlighted in red (EC): the hippocampal body (HB), hippocampal head $(\mathrm{HH})$, insula $(\mathrm{IN})$, and inferior and middle temporal neocortex (TP). Lower row: Plots of a single-parameter classification (T2r) for the subregions EC, $\mathrm{HH}$, and $\mathrm{HB}$. The axes correspond to the largest orthogonal eigenvectors found by the principal component analysis. Patients $(n=54)$ with Alzheimer disease $(A D)$ and mild cognitive impairment $(\mathrm{MCl})$ and healthy controls (CTRLs) within the $\mathrm{EC}(A), \mathrm{HH}(B)$, and $\mathrm{HB}(C)$ were classified into 4 (CTRL/MCI, CTRL, MCl, and AD/MCl) clusters (AD [red], $\mathrm{MCl}$ [blue], and CTRLs [green]). Cluster selectivity reached 0.87 for $A, 0.84$ for $B$, and 0.86 for $C$. The strongest effects of changes within the macromolecular matrix were detected within the mesiotemporal lobe. and CTRLs $(28.67 \pm 1.49$ versus $29.50 \pm$ $0.7)$. The CERAD-NAB mean score was $51.71 \pm 19.01$ for patients with $\mathrm{AD}, 76.62 \pm$ 15.47 for those with $\mathrm{MCI}$, and $90.56 \pm$ 15.82 for the CTRLs, and it differed between patients with $\mathrm{AD}$ and CTRLs, between patients with $\mathrm{AD}$ and $\mathrm{MCI}$, and between patients with MCI and CTRLs $(P<.05)$. The verbal fluency and word list delayed-recall subitems of the CERAD-NAB further discriminated between patients with $\mathrm{MCI}$ and CTRLs $(P<.05)$.

The CERAD-NAB test parameters are listed in the Table, with the participant demographic data.

\section{Individual Classification}

For individual classification, the averaged left and right magnetization transfer values between corresponding VOIs were used for further processing: A concordance of 0.92 ( 50 of 54 subjects) with the CERAD was achieved by the combination of subset 2 (kf, kr, T2r, F, T2f) in the entorhinal cortex. The sensitivity and specificity for the discrimination of AD from CTRL reached 1; for the discrimination of AD from MCI, 1 (95\% CI, 0.78-1) and 0.94 (95\% CI, 0.71-1), with a positive predictive value of 0.95 and a negative predictive value of 1 . For the discrimination between MCI versus CTRLs, the sensitivity was 0.83 (95\% CI, $0.58-0.95)$, the specificity was 0.86 (95\% CI, 0.63-0.96), the positive predictive value was 1 , and the negative predictive value was 0.86 . The hippocampal head (selectivity of 0.83 ), hippocampal body (selectivity of 0.9), insula (selectivity of 0.73 ), and the inferior and middle temporal neocortex (selectivity of 0.83 ) contributed less to the classification and added no further information. Discrepancies between the magnetization transfer-based classifi-

\section{RESULTS}

\section{Clinical Classification of Patients with AD and $\mathrm{MCI}$ and CTRLs}

Patients with AD and MCI and the CTRLs did not differ significantly in terms of mean age (CTRLs, $71.61 \pm 9.2$ years; patients with MCI, $70.83 \pm 10.1$ years; and patients with $\mathrm{AD}, 70.39 \pm 9.9$ years) or educational status (CTRLs, $12.78 \pm 3.11$ years; patients with $\mathrm{MCI}, 11.28 \pm 1.99$ years; and patients with $\mathrm{AD}, 11.00 \pm 3.30$ years). The global cognitive functioning level, as indicated by the Mini-Mental State Examination, differed significantly between CTRLs and patients with $\mathrm{AD}(29.50 \pm 0.7$ versus $24.56 \pm 3.45$; $P<.05)$ and between patients with MCI and AD $(28.67 \pm 1.49$ versus $24.56 \pm 3.45 ; P<.05)$ but not between patients with MCI cation and the clinical and neuropsychological classifications were detected in 4 of the 18 subjects with MCI. Three were misclassified as CTRLs, and 1, as AD. Among these 4 patients, the CTRLs (misclassified as patients with MCI) had higher CERAD scores $(95,85$, and 78$)$ than the mean MCI score $(76.62 \pm 15.47)$. One was younger and 2 were older $(70,72$, and 81 years) than average $(70.83 \pm 10.1$ years $)$. The patients with MCI misclassified as those with $\mathrm{AD}$ had the lowest CERAD (61) and Mini-Mental State Examination (25) scores among the MCI group and were older than average ( 79 years). Omission of the T2f parameter in subset 1 (kf, kr, T2r, F, T2f) resulted in a concordance of 0.91 (49 of 54 patients) with mis- 


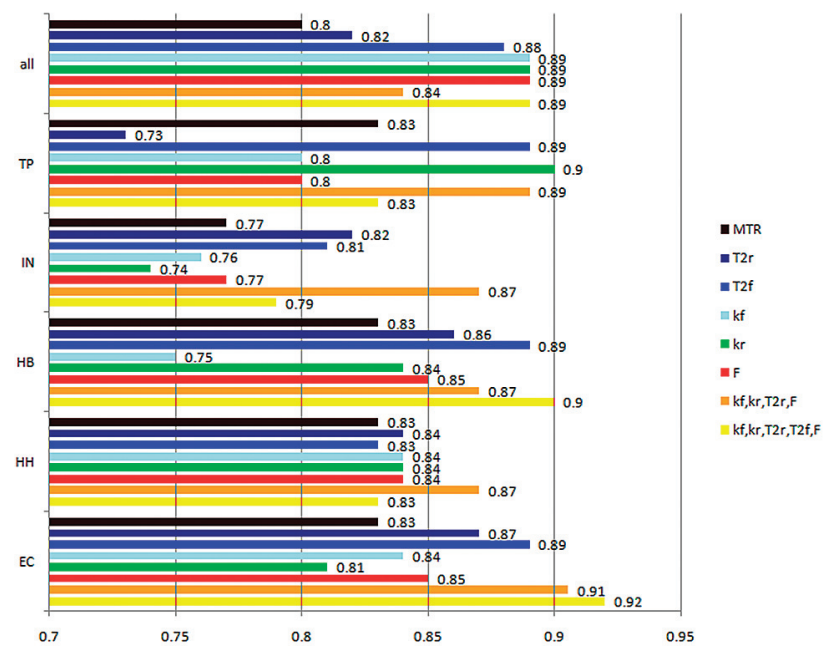

FIG 2. Cluster selectivity for the mMT parameters incorporated into the classification of the 5 VOls and parameters (MTR, T2r, T2f, F, kr, kf, subset 1 and subset 2). The selectivity of the classification reached 0.92 (50 of 54 subjects) for subset 2 in the entorhinal cortex. The multiparameter classification of subset 1 revealed significantly improved selectivity of the patients with $A D$ and $M C l$ and CTRLs compared with MTR.

classification of another patient with MCI having AD. Notably, this patient had the second lowest CERAD score and was the oldest subject ( 84 years).

With the best single parameter classifications, by using the mMT of the free (T2f), 6 subjects (selectivity of 0.89 ), and the restricted pool only (T2r), 7 subjects (selectivity of 0.87 ) were misclassified in the entorhinal cortex. Bootstrap analysis revealed a negative correlation between T2f and T2r (correlation coefficient, -0.07). For MTR, the clustering was less effective and resulted in a discrepancy in 9 patients with MCI who were all misclassified as having AD. The clustering based on MTR values resulted in a selectivity of 0.83 in the entorhinal cortex, hippocampal head, hippocampal body, and the inferior and middle temporal neocortices and 0.77 in the insula. For the essential discrimination between $\mathrm{AD}$ and MCI, MTR reached a lower specificity of 0.5 compared with 0.94 and a positive predictive value of 0.67 compared with 0.95 for mMT, while sensitivity and NPV remained equal. Patients with MCI who were misclassified as having AD by MTR revealed a nonsignificant trend toward increased age (mean, $74.1 \pm 9.8$ years) compared with the 9 other subjects with MCI (mean, $67.5 \pm 9.7$ years). There were no differences in Mini-Mental State Examination and CERAD total scores for both subgroups (mean, $28.4 \pm 1.8$ versus $28.9 \pm 1.16 ; 76.6 \pm 9.5$ versus $76.7 \pm 11.3$; not significant). The combination of all VOIs for MTR and mMT failed in improving the quality of the classification ( 0.84 for subset 1 and 0.89 for subset 2, 0.82 for T2r, 0.88 for T2f, 0.89 for kr and kf, 0.89 for F, and 0.8 for MTR). The results of the automated classification are summarized in Fig 2.

\section{DISCUSSION}

In this study, we have demonstrated the feasibility and potential value of mMT imaging techniques for the classification of patients with de novo $\mathrm{AD}$ and $\mathrm{MCI}$. The neuropsychological functions according to the CERAD-NAB have been used as a clinical reference for patient stratification at first-time referral to a memory clinic because neuropsychological measures are widespread, available, and accurate for identifying subjects in the prodromal phase of AD. ${ }^{31}$ This study revealed 3 important findings: 1) Automatic classification differentiated patients with $\mathrm{AD}$ and $\mathrm{MCI}$ according to the macromolecular tissue composition in the entorhinal cortex with a sensitivity of 1.00 and a specificity of 0.94 by a combination of all key model parameters calculated with mMT. 2) mMT yielded a higher specificity compared with MTR in the discrimination of subjects with AD and MCI. 3) Predominant effects due to changes in the macromolecular tissue composition were detected in the entorhinal cortex.

The integration of the entorhinal cortex as a core structure in the evolution of $\mathrm{AD}$ and the assignment of hyperellipsoidal clusters resulted in an improved classification of $\mathrm{AD}$ and $\mathrm{MCI}$ compared with previous studies that used mMT in the hippocampus. ${ }^{15,30}$ This finding correlates well with histopathologic studies that used the Braak and Braak ${ }^{32,33}$ and Braak et $\mathrm{al}^{34}$ classifications and assumed that the highest changes within the macromolecular matrix take place in the entorhinal cortex during the early stages of cognitive decline. Transsynaptic spread of $\tau$ pathology from the entorhinal cortex to the hippocampus along the perforant pathway is a potential mechanism associated with molecular alterations responsible for aging and $\mathrm{AD}^{35}$ that are detectable by mMT. The relative size of the restricted proton pool, the magnetization exchange rates, and the relaxation time of the restricted pool can be considered markers of restricted protons within such macromolecular structures. Because pathologic accumulations of soluble and nonsoluble proteins precede cell death, alterations in the local composition of macromolecules may be more relevant than local concentration or atrophy. The advantage of extending the magnetization transfer technique from a single parameter (MTR) to a model-based multiparameter approach offers to separately quantify the presence and amount of macromolecules and to investigate the coupling characteristics of protons by modeling depositions and interactions. mMT thus may aid in classifying different types of macromolecules in contrast to MTR, which does not discriminate between amount and type of macromolecules. Of note, the omission of T2f (as an indirect indicator for aging and atrophy due to the load of interstitial water) in subset 1 resulted in a reduction of the cluster selectivity in the entorhinal cortex from 0.92 to 0.91 , related to the misclassification of the oldest patient enrolled into the study as having MCI. $\mathrm{T} 2 \mathrm{f}$ should, therefore, be considered as a fundamental parameter that adds complementary information for appropriate clustering in selected cases.

Previous studies have reported either increases ${ }^{15}$ or decreases ${ }^{16}$ of the parameter $F$, which may reflect an increased deposit of macromolecules or an increased load of interstitial water. Therefore $\mathrm{T} 2 \mathrm{f}$ may be further incorporated as a control parameter for such effects.

The methodology used in this study is diverging from learning algorithms that are frequently used for the detection of structural abnormalities: Support vector machines, as are currently frequently used for the classification of T1-weighted datasets in $\mathrm{AD}$ and MCI, use a supervised learning approach that incorporates a set of training objects whose membership to a certain class is known a priori. Otherwise, data that have not been addressed for 
learning in advance maybe misclassified. Support vector machinebased methods reported a sensitivity and specificity to discriminate controls from $\mathrm{AD}$ of $89 \%$ and $94 \%$ and controls from MCIconverters of $89 \%$ and $80 \% .{ }^{36}$ Clustering, in contrast, is a datadriven unsupervised approach, that does not need a priori knowledge about the membership of the objects but classifies them by finding similarities in the data. With unsupervised techniques, such as the Gustafson-Kessel algorithm, it is possible to learn larger and more complex models than with supervised approaches. This may, despite the relatively small cohort enrolled in this study, explain the improved specificity of a multiparameter classification (subsets 1 and 2) compared with MTR and T2r, T2f, $\mathrm{F}, \mathrm{kr}, \mathrm{kf}$ as solitary input parameters. A potential bias due to $\mathrm{T} 2^{*}$ effects in the entorhinal cortex can be excluded because $\mathrm{T} 2^{\star}$ effects may be relevant predominantly during the readout period of the sequence (which is the same for all magnetization transfer frequency offsets).

One limitation of this study is its cross-sectional character, focusing on a neuropsychological classification at first referral. Neuropsychological classification is well-established and broadly available as a clinical reference standard; and CERAD scores, ${ }^{31}$ alone or in combination with Mini-Mental State Examination $<26$, have been demonstrated to reliably predict future trajectories of cognitive decline. ${ }^{37}$ The conversion from MCI to AD, however, cannot be directly determined from our data. Further longitudinal clinical analyses are necessary to prove the clinical stratification of the different cohorts at the point of access that has been used as a reference in this study. We aim to incorporate in vitro studies, along with larger longitudinal clinical studies, of postmortem brains to determine whether (soluble) plaques, fibrils, or accumulated microglia cells are causative for alterations in the local composition of macromolecules.

\section{CONCLUSIONS}

In summary, we demonstrated that model-based magnetization transfer imaging by using a subset of modeled parameters detects macromolecule-related alterations in the mesial temporal lobe (dominantly in the entorhinal cortex) and that an individual classification based on the mMT may improve the classification of patients with de novo $\mathrm{AD}$ and MCI compared with MTR. In the future, MR imaging-based neuroimaging approaches might incorporate advantages of protocols targeted against focal differences in brain anatomy (with respect to the detection of gray matter loss) and magnetization transfer effects regarding the opportunity to classify macromolecules according to the strength of coupling to the environment.

\section{ACKNOWLEDGMENTS}

We thank the radiographers M. Mordasini and. C. Stuker for their excellent technical assistance.

Disclosures: Roland Wiest-RELATED: Grant: Kamillo-Eisner Foundation,* Comments: private funding (CHF 77000), UNRELATED: Grants/Grants Pending: Scherbarth Foundation. * Martinus Hauf_UNRELATED: Grants/Grants Pending: Swiss National Science Foundation grant 33CM30-124089.* Gerhard Schroth-UNRELATED: Grants/Grants Pending: Swiss National Science Fond, * Siemens-Bayer Scientific Cooperation.* Martin Zbinden—RELATED: Grant: Kamillo-Eisner.* Claus Kiefer-RELATED: Grant: Kamillo-Eisner. *Money paid to the institution.

\section{REFERENCES}

1. Roe CM, Fitzpatrick AL, Xiong C, et al. Cancer linked to Alzheimer disease but not vascular dementia. Neurology 2010;74:106-12

2. Dickerson BC, Stoub TR, Shah RC, et al. Alzheimer-signature MRI biomarker predicts $\mathrm{AD}$ dementia in cognitively normal adults. Neurology 2011;76:1395-402

3. Haller S, Lovblad KO, Giannakopoulos P. Principles of classification analyses in mild cognitive impairment (MCI) and Alzheimer disease. J Alzheimers Dis 2011;26(suppl 3):389-94

4. Chupin M, Hammers A, Liu RS, et al. Automatic segmentation of the hippocampus and the amygdala driven by hybrid constraints: method and validation. Neuroimage 2009;46:749-61

5. Calvini P, Chincarini A, Gemme G, et al. Automatic analysis of medial temporal lobe atrophy from structural MRIs for the early assessment of Alzheimer disease. Med Phys 2009;36:3737-47

6. Whitwell JL, Shiung MM, Przybelski SA, et al. MRI patterns of atrophy associated with progression to $A D$ in amnestic mild cognitive impairment. Neurology 2008;70:512-20

7. Chincarini A, Bosco P, Calvini P, et al. Local MRI analysis approach in the diagnosis of early and prodromal Alzheimer's disease. $\mathrm{Neu}$ roimage 2011;58:469-80

8. Jack CR Jr. Alzheimer disease: new concepts on its neurobiology and the clinical role imaging will play. Radiology 2012;263:344-61

9. Kabani NJ, Sled JG, Shuper A, et al. Regional magnetization transfer ratio changes in mild cognitive impairment. Magn Reson Med 2002; 47:143-48

10. van der Flier WM, van den Heuvel DM, Weverling-Rijnsburger AW, et al. Magnetization transfer imaging in normal aging, mild cognitive impairment, and Alzheimer's disease. Ann Neurol 2002;52: 62-67

11. Ropele S, Schmidt R, Enzinger C, et al. Longitudinal magnetization transfer imaging in mild to severe Alzheimer disease. AJNR Am J Neuroradiol 2012;33:570-75

12. Stanisz GJ, Yoon RS, Joy ML, et al. Why does MTR change with neuronal depolarization? Magn Reson Med 2002;47:472-75

13. Sled JG, Pike GB. Quantitative imaging of magnetization transfer exchange and relaxation properties in vivo using MRI. Magn Reson Med 2001;46:923-31

14. Stanisz GJ, Odrobina EE, Pun J, et al. T1, T2 relaxation and magnetization transfer in tissue at 3T. Magn Reson Med 2005;54:507-12

15. Kiefer C, Brockhaus L, Cattapan-Ludewig K, et al. Multi-parametric classification of Alzheimer's disease and mild cognitive impairment: the impact of quantitative magnetization transfer MR imaging. Neuroimage 2009;48:657-67

16. Giulietti G, Bozzali M, Figura V, et al. Quantitative magnetization transfer provides information complementary to grey matter atrophy in Alzheimer's disease brains. Neuroimage 2012;59:1114-22

17. McKhann G, Drachman D, Folstein M, et al. Clinical diagnosis of Alzheimer's disease: report of the NINCDS-ADRDA Work Group under the auspices of Department of Health and Human Services Task Force on Alzheimer's Disease. Neurology 1984;34:939-44

18. Petersen RC. Mild cognitive impairment as a diagnostic entity. J Intern Med 2004;256:183-94

19. Jeon YH, Sansoni J, Low LF, et al. Recommended measures for the assessment of behavioral disturbances associated with dementia. Am J Geriatr Psychiatry 2011;19:403-15

20. McKhann GM. Changing concepts of Alzheimer disease. JAMA 2011;305:2458-59

21. MacFall JR, Wehrli FW, Breger RK, et al. Methodology for the measurement and analysis of relaxation times in proton imaging. Magn Reson Imaging 1987;5:209-20

22. Haase A. Snapshot FLASH MRI: applications to T1, T2, and chemical-shift imaging. Magn Reson Med 1990;13:77-89

23. Deichmann R, Adolf H, Kuchenbrod E, et al. Compensation of diffusion effects in T2 measurements. Magn Reson Med 1995;33:113-15

24. Deichmann R, Adolf H, Noth U, et al. Fast T2-mapping with snapshot flash imaging. Magn Reson Imaging 1995;13:633-39

25. Bobinski M, de Leon MJ, Wegiel J, et al. The histological validation 
of post mortem magnetic resonance imaging-determined hippocampal volume in Alzheimer's disease. Neuroscience 2000;95: $721-25$

26. Mosconi L, Brys M, Glodzik-Sobanska L, et al. Early detection of Alzheimer's disease using neuroimaging. Exp Gerontol 2007;42: $129-38$

27. Fennema-Notestine C, Hagler DJ Jr, McEvoy LK, et al. Structural MRI biomarkers for preclinical and mild Alzheimer's disease. Hum Brain Mapp 2009;30:3238-53

28. Walhovd KB, Fjell AM, Dale AM, et al. Multi-modal imaging predicts memory performance in normal aging and cognitive decline. Neurobiol Aging 2010;31:1107-21

29. Teslic L, Hartmann B, Nelles O, et al. Nonlinear system identification by Gustafson-Kessel fuzzy clustering and supervised local model network learning for the drug absorption spectra process. IEEE Trans Neural Netw 2011;22:1941-51

30. Ridha BH, Tozer DJ, Symms MR, et al. Quantitative magnetization transfer imaging in Alzheimer disease. Radiology 2007;244:832-37

31. Paajanen T, Hanninen T, Tunnard C, et al. CERAD neuropsychological battery total score in multinational mild cognitive impairment and control populations: the AddNeuroMed study. J Alzheimers Dis 2010;22:1089-97

32. Braak H, Braak E. Neuropathological stageing of Alzheimer-related changes. Acta Neuropathol 1991;82:239-59

33. Braak H, Braak E. Morphological criteria for the recognition of Alzheimer's disease and the distribution pattern of cortical changes related to this disorder. Neurobiol Aging 1994;15:355-56, discussion $379-80$

34. Braak H, Thal DR, Ghebremedhin E, et al. Stages of the pathologic process in Alzheimer disease: age categories from 1 to 100 years. J Neuropathol Exp Neurol 2011;70:960-69

35. Stranahan AM, Mattson MP. Selective vulnerability of neurons in layer II of the entorhinal cortex during aging and Alzheimer's disease. Neural Plast 2010:2010;108190

36. Cuingnet R, Gerardin E, Tessieras J, et al. Automatic classification of patients with Alzheimer's disease from structural MRI: a comparison of ten methods using the ADNI database. Neuroimage 2011;56: $766-81$

37. Wilkosz PA, Seltman HJ, Devlin B, et al. Trajectories of cognitive decline in Alzheimer's disease. Int Psychogeriatr 2010;22:281-90 